\title{
Essentials in the diagnosis of postoperative myocardial lesions similar to or unrelated to rejection in heart transplant
}

\author{
Costel Dumitru ${ }^{1}$, Ancuta Zazgyva ${ }^{1,2}$, Adriana Habor ${ }^{1,3}$, Ovidiu Cotoi ${ }^{1,2,4}$, Horațiu \\ Suciu $^{1,5}$, Carmen Cotrutz ${ }^{6}$, Bogdan Grecu ${ }^{6 *}$, Ileana Anca Sin ${ }^{1,2,7}$
}

1. George Emil Palade University of Medicine, Pharmacy, Science, and Technology of Targu Mures, Romania; 2. Department of Cell and Molecular Biology; 3. Department of Physiology; 4. Department of Pathophysiology; 5. Institute of Emergency for Cardiovascular Diseases and Transplantation of Targu Mures, Department of Surgery V; 6. Department of Cell and Molecular Biology, Grigore T Popa University of Medicine and Pharmacy, Romania; 7. Department of Anatomopathology, Mureș County Emergency Clinical Hospital, Romania

\begin{abstract}
Background, objectives: Histological diagnosis of control biopsies in patients with heart transplant represents a significant step of monitoring, with a great influence on adjusting immunosuppressive treatment. Histological lesions are usually related to ischemia and reperfusion, with varying degrees of intensity. This study aimed to highlight the most important aspects of the histological diagnosis and differential diagnosis of postoperative myocardial lesions associated or unrelated to rejection in heart transplant. Materials and Methods: This retrospective study involved 53 patients who received cardiac transplant between 2000 and 2017. Patients were monitored by lesion quantification of endomyocardial biopsies, with diagnoses established based on biopsy material in the early, medium and late post-transplant periods. Hematoxylin eosin, Masson's trichrome, and Van Gieson stains were used; immunohistochemical determinations used CD4, CD20, CD45, CD68, HLA-DR, VEGF and CD31. Results: Ischemia and reperfusion lesions were diagnosed on all biopsies in the first 6 weeks post-transplant. Nine cases of the Quilty effect were identified, and in 12 cases, the biopsies were performed on the same spot as previous biopsies. A significant number of transplanted patients presented cytomegalovirus that was difficult to diagnose on endomyocardial biopsies. Conclusions: The detailed study of ischemia and reperfusion lesions, as well as of changes un-related to rejection becomes a major objective in the short, medium and late post-transplant period. Overdiagnosis of rejection induces changes of the immunosuppressive therapeutic protocol, with alarming repercussions on
\end{abstract}

\footnotetext{
* Corresponding author: Vasile Bogdan Grecu, Department of Cell and Molecular Biology, Grigore T Popa University of Medicine and Pharmacy, Iași, Romania. E-mail: grecu_bogdan@ymail.com
} 
cytomegalovirus reactivation, and risks of potentiating inflammation, myocyte destruction and the recurrence of disorders related to both inducing and aggravating heart failure.

Keywords: ischemia, reperfusion, heart transplant, Quilty effect

Received: $7^{\text {th }}$ August 2020; Accepted: $1^{\text {st }}$ March 2021; Published: $7^{\text {th }}$ April 2021

\section{Introduction}

Although cardiac transplantation substantially improves the survival and quality of life of patients with the most advanced stages of heart failure, most recipients will present complications in the early, mid- or late post-transplant periods (1). The most common histological lesions found in all patients with heart transplant are those related to ischemia and reperfusion, with varying degrees of intensity. These can easily be diagnosed within the first 6 weeks post-transplant, but may persist for up to three months after surgery. It is sometimes difficult to establish a differential diagnosis between these lesions and acute cellular rejection due to the common manifestations of lesions. The Quilty effect - which appears post-transplant in a relatively small number of patients, predominantly male - is a lesion with not yet fully elucidated appearance and effects on the evolution of the graft over time; some authors suggest its association with graft rejection or the prefiguration of an episode of rejection (2).

Patients undergo repeated control biopsies, with additional biopsies performed after episodes of rejection - thus, in several cases, the re-biopsy is made in an area that has been previously biopsied, which leads to visualizing a scar and a residual inflammatory infiltrate. The adipose tissue (a normal component of the myocardium) can be found on myocardial biopsies, including dilated vessels or showing mesothelial fragments which denote perforation of the right ventricle. Perimyocytic interstitial fibrosis occurs as a result of increased doses of the immunosuppressant, while myocyte calcifications, although rare lesions, may be caused by transient hypercalce- mia. At 1-2 months after transplantation and even more obviously after 3 years, a longer period of ischemia can lead to - sometimes advanced myocardial hypertrophy. A major cause of death may be the appearance of infections, the risk of their occurrence being mainly due to immunosuppression. Quite frequently, myocardial biopsies show Cowdry viral inclusions characteristic of the presence of cytomegalovirus, or cell inclusions belonging to other types of viruses.

There are also other manifestations of lesions sometimes accidental - such as foreign-body giant cell reactions, relapse of the initial disease, valve fragments or intravascular lymphocytes, which must be reported to clinicians in order to eliminate the risk of secondary pathology with serious consequences for the evolution of patients with heart transplant. A correct diagnosis is essential - starting from the biopsy tissue sample, using standard staining and immunohistochemistry (which has recently been associated with serological markers to monitor patient progress) - to identify non-rejection histological lesions, among which the most common are ischemia and reperfusion, but also those less frequently found, since many of these can easily be confused with rejection. This highlights the significance of knowing clear histological characteristics of these lesions, so that they can be identified without over-diagnosing a rejection reaction.

This study aimed to highlight the most important aspects of the histological diagnosis and differential diagnosis of postoperative myocardial lesions associated or unrelated to rejection in heart transplant, which represents a significant step in the monitoring of heart transplant patients and has a great influence on adjusting immunosuppressive treatment. 


\section{Materials and Methods}

Our retrospective study on the diagnosis of non-rejection-related or rejection-related postoperative myocardial lesions was performed on 53 patients who received cardiac transplant between 2000 and 2017 in the Clinic of Cardiovascular Diseases, Institute of Emergency for Cardiovascular Diseases and Transplantation (IUBCVT) of Târgu Mureș. All subjects gave their informed consent before they participated in the study, which was conducted in accordance with the Declaration of Helsinki, and the protocol was approved by the Ethics Committee of IUBCVT (305 B/15.07.2016).

The patients were regularly observed according to a pre-established protocol, monitored by lesion quantification of endomyocardial biopsies (EMB). Endomyocardial biopsy was performed according to international guidelines for medical practice in transplantation. The pathological monitoring report bulletin always mentions the difference regarding the evolution of the pathological manifestations between the previous and the present biopsy result. Thus, the patient is constantly monitored in his/ her evolution from one biopsy to another.

For the biopsies, the used approach was through the internal jugular vein for the right side of the right ventricular septum (method taken from US interventional cardiology centers). To avoid embolism, infusion was used in all arterial sheaths and anticoagulants were used. Fluoroscopy guidance provided the operator with more information about the level of the biopsy. Another approach was using echocardiography without fluoroscopy, when intra-cardiac tissue was targeted. CT or MRI assessments were useful to evaluate the angle between the ventricular septum and the superior or inferior vena cava. MRI provided useful information when localizing focal lesions for biopsies. The most common adverse event during biopsy was myocardial perforation, without major clinical implications, because the pericardium has numerous areas of postoperative adhesion. Echocardiography was always used when there was myocardial perforation. The risk of pneumothorax was avoided by using an approach in a higher area of the internal jugular vein. Of course, EMB has its associated risks, but they depend on the patient's condition and reactivity and the professionalism of the person who performs it.

The harvested material was fixed in formalin for histological determinations, in glutaraldehyde when there was the possibility of an examination in electron microscopy, and was preserved by freezing for immunohistochemistry or for PCR techniques, especially for viral determinations. For patients surviving over 5 years, the number of these biopsies reaches a significant value, with over 600 biopsies performed for the studied cases. The diagnosis of the lesions was established based on the assessment of material obtained from EMB performed according to the protocol of the Clinic in the early post-transplant period, as well as in the medium and late period. Histopathological examination was performed on sections stained with hematoxylin eosin, Masson's trichrome, and Van Gieson stains, while immunohistochemical determinations used CD4, CD20, CD45, CD68, HLA-DR, VEGF1, and $\mathrm{CD} 31$.

All cases with the Quilty effect were analyzed both histologically and immunohistochemically, at the endocardial level and the subendocardial myocyte level. All biopsies with adipocytes with or without mesothelium were analyzed, identifying ventricular perforation when it was evident. During histological analysis, cases of perimyocytic fibrosis, fibrotic scarring, myocardial hypertrophy and presence of cytomegalovirus (CMV) granulomas were also highlighted.

The small intra-myocardial vessels at the level of the endothelial and intima were additionally analyzed, mentioning the possible changes found in 
the lumen and wall thickness, and indicating the presence or absence of perivascular or intraluminal monocytes.

The statistical analysis included both descriptive statistics (frequency, percentage) and inferential statistics. Fischer's test was applied in order to determine the association between the qualitative variables, with a significance threshold chosen as a p-value of 0.05. Statistical analysis was performed using the trial version of GraphPad Prism.

\section{Results}

The mean age at heart transplant was 40.72 years, with a majority of male patients (84.91\%). The mean age of the donors was 31.92 years, again, with the majority being male (66.67\%). The diagnosis on hospital admission was that of heart failure, most cases being classified as NYHA stage $4(60.40 \%)$. The majority of patient presented dilated cardiomyopathy (98.1\%), while the diagnoses of myocardial infarction and pulmonary hypertension were also frequent (49.06\% and $35.85 \%$ of cases respectively).

There were no fragments of tendinous cords or tricuspid valve in any of the cases. Myocytes with coagulation necrosis were found especially on restricted subendocardial areas - these lesions were reduced on the initial biopsy and more numerous on the biopsy material obtained in the mid and late periods after transplantation. In three cases, post-transplant micro-infarcts with small areas of neovascularization and lax connective tissue were reported around the affected area. The appearance of these areas required a differential diagnosis with the areas of coagulation necrosis due to prolonged ischemia occurring during the re-implantation of the heart or from the resuscitation period needed to resume the function of the transplanted heart.

Ischemia and reperfusion lesions were diagnosed on all biopsies performed in the first 6 weeks post-transplant. In some instances, the slightly atypical appearance of the lesions in cases of prolonged ischemia was difficult to differentiate from an incipient acute cellular rejection. Furthermore, in three of the cases where artifacts were noted (such as tissue stretching during sample harvesting), associated with the presence of interstitial edema, the distinction from a humoral rejection was also difficult to establish. In case with a clear diagnosis of lesions due to ischemia and reperfusion, subendocardial coagulation necrosis of several myocytes was noted, as well as a small number of monocytes, and even rare neutrophils (figure 1).

Using van Gieson's stain, we were able to highlight the degenerated myofibrils that appeared homogeneous and pale, devoid of nucleus. At 3 to 4 weeks post-transplantation, the inflammatory infiltrate was more abundant, but also polymorphic in appearance, with the presence of macrophages - this aspect could help distinguish an ischemia lesion from an acute rejection, as in the latter a diffuse or nodular lymphocyte infiltrate is predominant. Biopsies obtained between 6 and 8 weeks after transplantation showed areas of necrosis with the appearance of microinfarcts in the process of organizing, with the disappearance of necrotic myocytes and proliferation of connective tissue. It should be noted that 8 patients in whom the diagnosis based on the first biopsies was that of similar lesions unrelated to rejection, with lesions that were particularly difficult to differentiate from a rejection (due to the presence of a larger number of lymphocytes in the polymorphous inflammatory tissue, showing CD4 positivity, with incipient endothelial lesions without the presence of perivascular lymphocytes, but weakly positive HLA and rare changes characteristic of premyocytolysis) developed acute cellular rejection with obvious vasculitis. For all cases with a Quilty effect, lesions of ischemia and early and late reperfusion were diagnosed, with the suspicion of an acute rejec- 


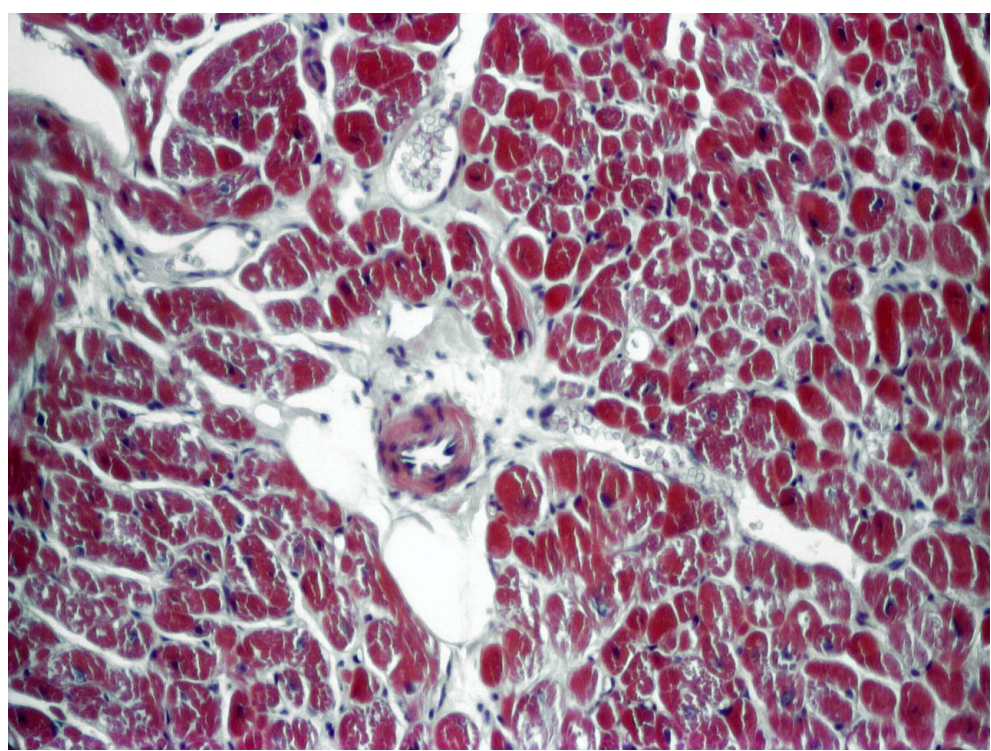

Fig. 1. Masson's trichrome stain - Endomyocardial biopsy - Ischemia and reperfusion lesion: premyocytolysis, endothelial lesions, minimal monocytic inflammatory infiltrate (200x)

tion. In the examined paraffin sections, 9 cases of the Quilty effect were identified, presenting the specific appearance of a dense endocardial lymphocytic infiltrate that, in most cases, extended to the underlying myocardium. The degree of extension varied from case to case, and, in two cases, the lymphocyte conglomerate affected only the endocardium. Three of the cases showed capillary vessels with a dilated lumen (figure 2a). Dense endocardial lymphocytic infiltrate was also detected using CD4, CD20, and CD68 antibodies. Thus, the Quilty effect was observed by the predominance of B lymphocytes immunoreactive to CD20 marker. T lymphocytes and macrophages in smaller numbers were detected by CD4 and CD68 antibodies, respectively, but not being sufficient to reveal an acute humoral rejection. Therefore, differential diagnosis was made with acute cellular rejection. In order to exclude the rejection, the tissue fragments were sectioned at several levels to highlight endocardial involvement. Additionally, the intermyocytic fibrous stroma and the presence of capillaries in the lymphocytic conglomerate further suggested the diagnosis of the Quilty effect, as well as the mixture of B and $\mathrm{T}$ lymphocytes (in acute rejection there is a predominance of T lymphocytes) (figure 2b, 3). On a number of 10 biopsies from 10 patients, performed 6 weeks after transplantation, VEGF expression was monitored in the endothelial cells of the small intramyocardial vessels, in the neovessels from the ischemic lesion areas, as well as in the fibroblasts from the microinfarct areas found post-transplant. In these cases where VEGF was positive, we considered that this endothelial growth factor has the capacity to induce physiological, compensatory angiogenesis in the ischemic lesions with coagulation necrosis (figure 4). In 12 cases we noted that the biopsies were performed on the same spot as previous biopsies. In two of these cases, probably after a re-biopsy closer to the date of the last intervention, a fibrinocruoric mass was noted, with areas of organized thrombus and with residual inflammatory lymphocyte and histiocytic material. The rest of the cases presented a focal, star-shaped scar (figure 5). The adjacent myocardium showed a 


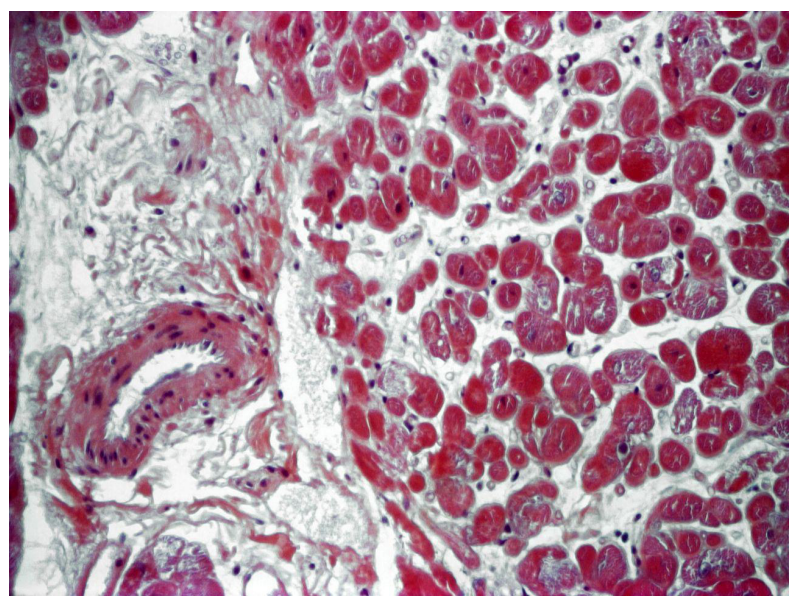

Fig. 2a. Masson's trichrome stain - Endomyocardial biopsy - Ischemia and reperfusion lesions.

Concentric thickening of the wall of an intramyocardial vessel (200x)

slight architectural disruption. The presence of the residual polymorphic infiltrate, sometimes of nodular appearance, is not related to a rejection reaction - this should be signaled, but does not need to be quantified.

On 26 of the biopsied cases we noted the presence of adipose cells in small numbers, or even in the form of adipose tissue fragments (figure 6). Fragments originating from the ventricu-

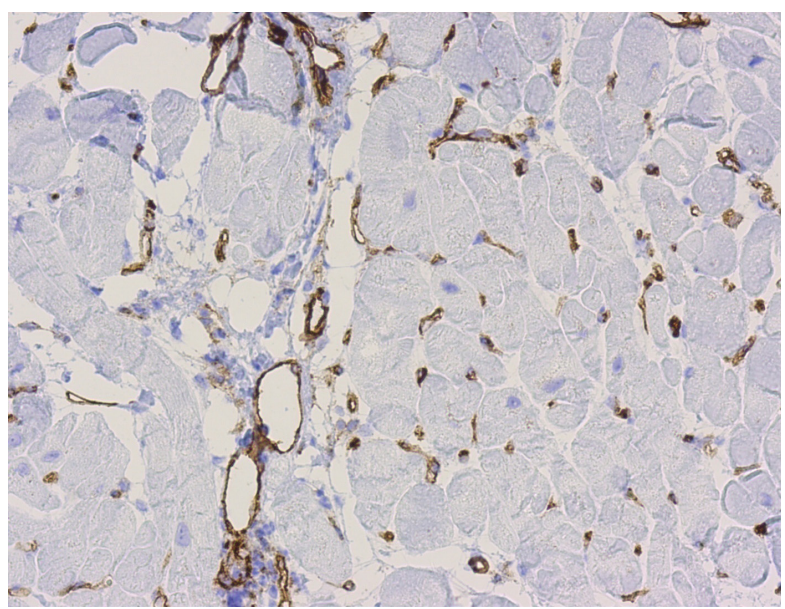

Fig. 3. CD31 - Endomyocardial biopsy - Acute humoral rejection. Intense positive stain of small intra-myocardial vessels $(200 x)$

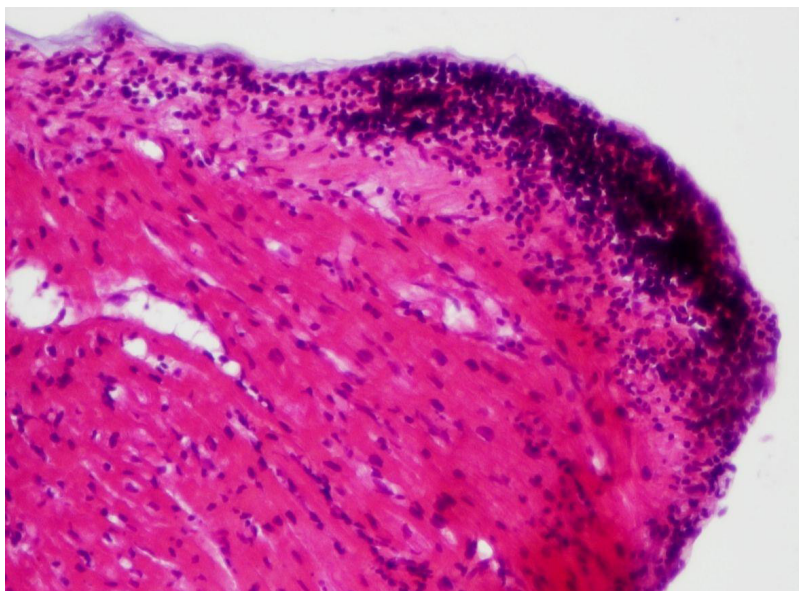

Fig. 2b. Hematoxylin eosin stain -

Endomyocardial biopsy - Quilty effect-lymphocytic inflammatory infiltrate in the endocardium and myocytes (200x)

lar wall have no pathological significance, but should be reported in the case of perforation of the ventricular wall when mesothelium is associated and there is a risk of a hemopericardium. Most of the times the risk is minimal due to the multiple areas of postoperative adherence of the pericardium.

A significant number of transplanted patients presented cytomegalovirus that was difficult to

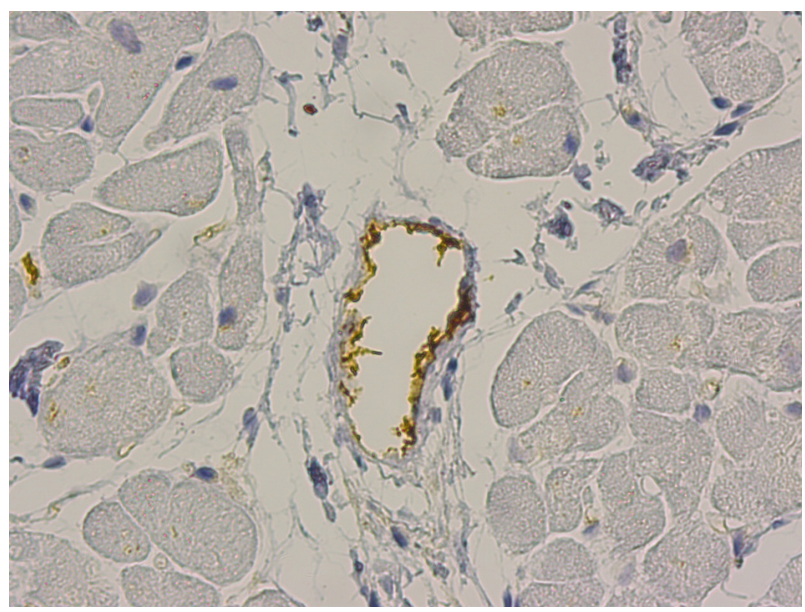

Fig. 4. VGF1 - Endomyocardial biopsy - Cytoplasmic and membranous immunohistochemistry stain marking the vascular endothelium (400x) 
diagnose on endomyocardial biopsies. Histological features consistent with CMV infection were observed in four cases, using monoclonal CMV antibody. Histologically, CMV granulomas consist of large cells, with characteristic Cowdry inclusions (figure 7a), surrounded by polymorphic inflammatory infiltrate and areas of myocytic necrosis. These inclusions were positive on CMV antibody, immunohistochemistry providing a certainty of CMV infection in these cases (figure 7b).

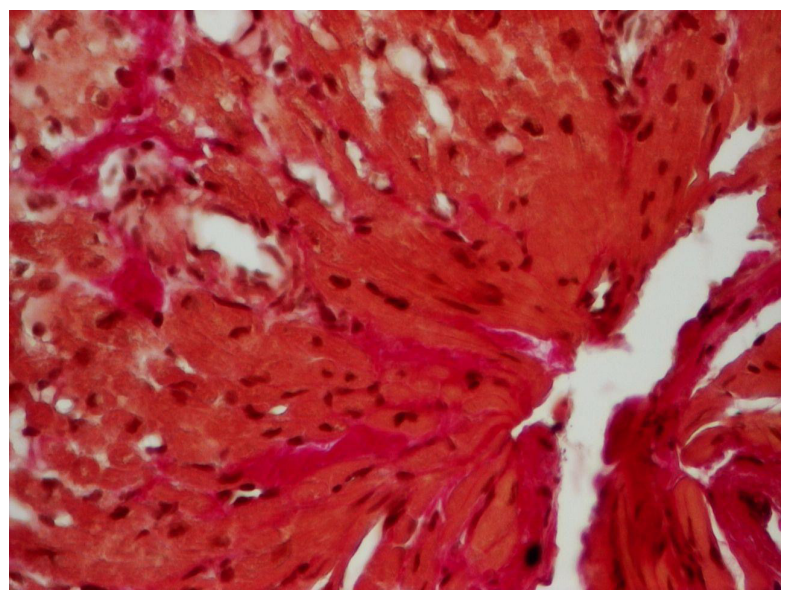

Fig. 5. Van Gieson's stain - Endomyocardial biopsy - Scarring fibrosis in the location of a previous biopsy (200x)

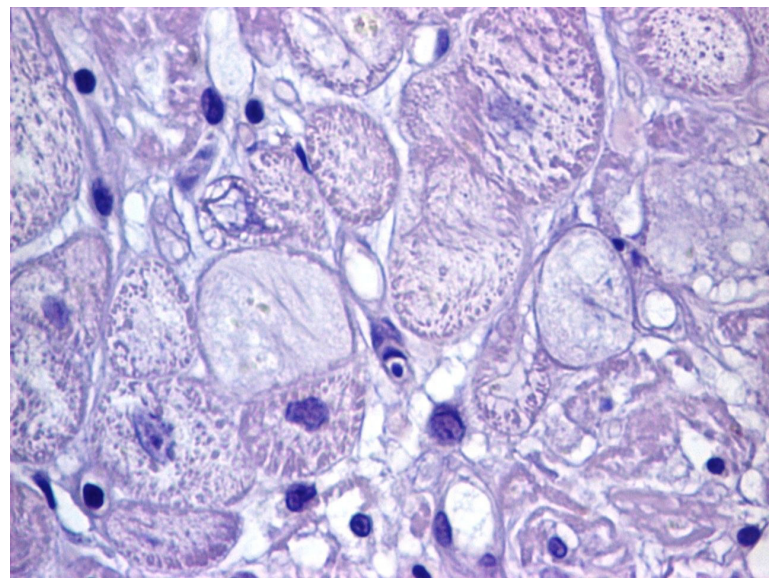

Fig. 7a. Hematoxylin eosin stain - Endomyocardial biopsy - CMV granuloma (detail). Large cell, with perinuclear halo and perinuclear intracytoplasmic inclusion (400x)

\section{Discussion}

The need for an early diagnosis of acute cellular and humoral rejection by way of a complex quantification of detected lesions, has led, since 1970, to the use of endomyocardial biopsies, which was included in the post-transplant monitoring protocol. The number of biopsy pieces varies from 3 to 6 tissue fragments, with sectioning made at 3 levels and, in most cases, processed within the first 24 hours (3). Artefacts may undesirably in-

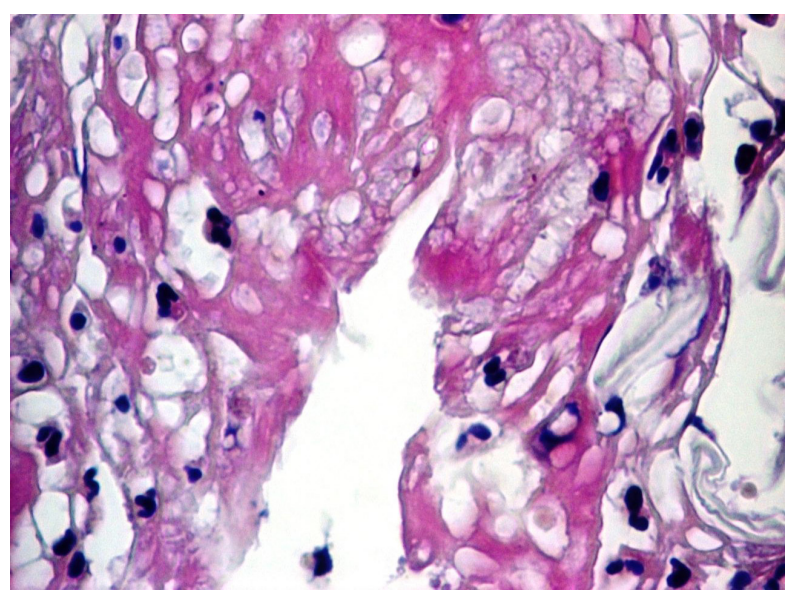

Fig. 6. Hematoxylin eosin stain - Endomyocardial biopsy - Adipocytes from the right ventricular wall, intra-cytoplasmic vacuolization $(400 x)$

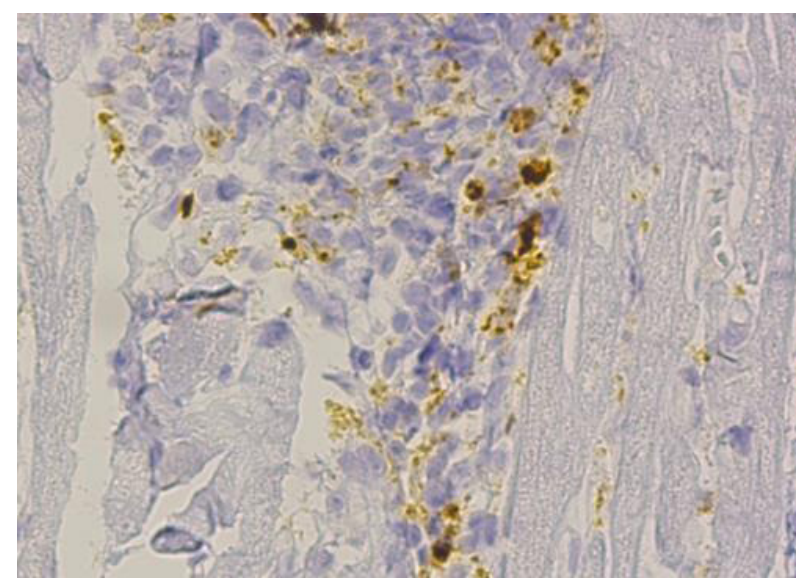

Fig. 7b. Hematoxylin eosin stain - Endomyocardial biopsy - CMV granuloma (detail). Large cell, with perinuclear halo and perinuclear intracytoplasmic inclusion (400x) 
fluence the diagnosis and therefore histological examination must reveal their presence, avoiding a possible confusion with an ischemia and reperfusion lesion or rejection. These include interstitial edema that appears in acute humoral rejection, but can also be observed in case of tissue stretching during harvesting, or the disorganization of myocytes at the edge of the biopsy area, with residual inflammatory infiltrate, denoting re-biopsy on an area from which myocardial tissue was collected previously, while the possibility of acute rejection is excluded based on the absence of a circumscribed, nodular infiltrate. The fibrosis that appears in these areas - in which a scar will be formed - is star-shaped, with digitiform penetration between the myocytes. By use of the van Gieson stain a differential diagnosis can be made between a re-biopsied area and a rejection. In addition, in the aforementioned areas, foreign-body giant cells or macrophages with hemosiderin pigment can be observed.

It is necessary to differentiate early ischemic changes from those detected in long-term survivors, who present with severe coronary atherosclerosis, with no signs of angina. These can easily be mistaken as acute rejection (4).

Regarding changes unrelated to rejection found in the studied patients, we can state that the Quilty effect, named after the first patient diagnosed with this type of lesion, can be detected either with no association with a rejection episode or coexisting with one, and can sometimes resorb after about 3 weeks even without immunosuppressive treatment. Its mechanism of appearance is not yet fully understood, but it may be linked to immunosuppressive treatment, and it seems that in the long term it does not lead to the appearance of lymphomas (5-7).

Although the pathogenesis of the Quilty effect has not yet been fully elucidated, recent studies have incriminated immunosuppressive treatment with cyclosporine, as well as Epstein Barr virus infection in the appearance of these lesions (8).
The fact that four of the patients for whom the Quilty effect was observed on previous biopsies had a small or moderate degree of acute rejection a month after the last biopsy does not show with certainty that these lesions represented early forms of cardiac rejection, as at the time the lesions were first observed there was no clinical evidence of acute rejection. In contrast, in a pediatric patient from our study, the Quilty effect was diagnosed concomitantly with a moderate cellular rejection associated with humoral rejection. According to international studies on large numbers of pediatric patients, these types of results suggest that the lesions found in the Quilty effect in children are correlated with vasculopathy and acute rejection (9). However, the literature specifically mentions the fact that these results are not conclusive and further investigations are needed to decipher the complex mechanisms of the possible causal relationship between the Quilty effect and rejection $(10,11)$. Regarding VEGF, studies have shown that it functions as a primary stimulus in the process of angiogenesis, a process whereby VEGF receptors stimulate signaling pathways that induce endothelial cell migration which have the ability to break down and remodel the extracellular matrix (12). This leads to an interesting possible study on the role of the different types of VEGF and of the two tyrosine kinase receptors, VEGFR-1 and VEGFR-2, in mediating endothelial survival signals and in post-transplantation ischemia and inflammation processes $(13,14)$.

Endothelial dysfunction plays an important role in the evolution of vascular lesions and the remodeling of vessels after myocardial revascularization. The main mechanisms involve the activity of metalloproteinases, the migration and proliferation of vascular smooth muscle cells, as well as the expression of pro-inflammatory cytokines (15). Recent data refer to circulating progenitor endothelial cells that present CD34 on their surface (similar to damaged endothelial 
cells from ischemic areas) and are captured by endothelial cells in the presence of the VEGF receptor located on the surface of these progenitor cells. Thus, the evolution of ischemic myocardial lesions depends on the circulating level of these progenitor cells.

The fibrosis commonly encountered on biopsies is perimyocytic and fine, and it appears shortly after immunosuppressive treatment. Fibrosis is more evident in patients treated with cyclosporine and occurs as a result of the high doses of immunosuppressants associated with ischemia and is influenced by the recipient's age (16). The use of next-generation immunosuppressants greatly reduces the risk of occurrence of interstitial fibrosis and its side effects $(17,18)$. In general, in patients in their mid-and - more frequently - late post-transplantation period, myocyte hypertrophy was noted either segmental or isolated on small groups of myocytes, seemingly related to the duration of the ischemia period and the areas of coagulation necrosis, after episodes of repeated rejection.

Infections remain a major cause of post-transplant morbidity and mortality. The factors that favor the occurrence of infections are related to: the comorbidities of the recipients or donors, trauma related to tissue harvesting and the moment of graft implantation, denervation, as well as the effects of the immunosuppressive treatment. Bacterial infections are common in the first three weeks after the transplant, and of the viral infections, cytomegalovirus remains the most common infectious agent. A first CMV infection in a seronegative recipient is accompanied by serious clinical manifestations, and the survival rate may be very low. Careful examination of the patient, serological analyses of the recipient and the donor, as well as the timely detection of the involvement of the virus in the induction or potentiation of a chronic rejection are paramount. Prophylactic treatment of seronegative patients who received a heart from seropositive donor with Ganciclovir or with specific immunoglobulin greatly reduces the risk of subsequent infection and the occurrence of chronic rejection (19, 20).

Sometimes the clinical manifestations of a CMV infection are confusing: some patients are asymptomatic, even if they are serologically positive, while others, on the contrary, have alarming clinical manifestations, such as disturbances of liver function, fever, thrombocytopenia, or even the presence of atypical lymphocytes.

The definitive diagnosis can be established by in situ hybridization. Serologically, during their post-transplantation evolution, approximately $70 \%$ of the survivors present $\mathrm{CMV}$, and it is assumed that immunosuppression favors the reactivation of the virus by blocking the action of $\mathrm{T}$ lymphocytes that can no longer control viral replication (21). Disrupting immunity by administering immunosuppressive treatment to seronegative recipients who received seropositive hearts affects the development of specific CMV immunity. A latent virus is influenced by inflammation caused by ischemia/ reperfusion, as well as by specific immunity $(22,23)$. Thus, CMV-specific immunization prior to transplantation becomes of major importance for CMV-seronegative recipients who will receive CMV-positive hearts, and recent studies aim to detect the immunosuppressive components with the greatest influence on post-transplant viral reactivation.

Toxoplasma gondii infection can be acquired from the seropositive donor or following reactivation of a latent infection $(24,25)$. For this ubiquitous microorganism, serology results show that more than $30 \%$ of adults have had a previous infection with this agent. Histologically, cysts of toxoplasma gondii appear in the myocardial cells, as well as a mixed inflammatory infiltrate, containing eosinophils. Differential diagnosis is usually made with micro-calcifications that can be found post-transplant. In our cases, the serological diagnosis was not associ- 
ated with a characteristic histological aspect for toxoplasma.

Undoubtedly, other microorganisms were also detected in our cases as in others presented in the literature, such as herpes viruses, Aspergillus, Pneumocystis carinii, mycobacteria, or the hepatitis $\mathrm{B}$ and $\mathrm{C}$ viruses. In certain cases, histological diagnosis is difficult to establish in a viral infection that is associated with inflammation and necrosis, especially when there are no microorganisms directly associated with the infection. Sometimes it is difficult to differentiate a virus-induced lesion from an acute rejection (26, 27). In these instances the results of the initial biopsy (with differential diagnosis difficulties) are compared with the results of subsequent biopsies, performed after specific treatment for the infection have already been administered. The persistence of an inflammatory infiltrate after administering anti-infective treatment is a sign of a rejection.

The long-term survival of patients with heart transplantation depends on the prevention or control of the immunological and non-immunological risk factors, the adequate adaptation of the immunosuppressive therapy and the modulation of the conventional immunosuppressive protocol in order to prevent episodes of acute cellular and humoral rejection, so as to prevent endothelial damage and avoid cardiac allograft vasculopathy. Thus, the detailed study of ischemia and reperfusion lesions, as well as that of all changes un-related to rejection, but which present the potential to influence the evolution towards this type of pathology, becomes a major objective in the short, medium and late post-transplant period.

\section{Conclusions}

The importance of histopathological diagnosis in monitoring the patients with cardiac transplantation was highlighted in our study by ob- serving and differentiating the lesions that mimic an acute cellular rejection (ischemia and reperfusion lesions, infections, Quilty effect) from the actual phenomena of cellular rejection, in order to avoid over-diagnosis, which automatically induces certain changes of the immunosuppressive therapeutic protocol.

Reactivation of CMV infection or the possibility of recurrence of other disease (such as giant cell myocarditis, amyloidosis, Chagas disease, sarcoidosis) which induce and could potentially aggravate heart failure, needs to be reported, so that the transplant team can intervene in a timely manner to treat the patient accordingly.

\section{Authors' contribution}

CD - Conceptualization, methodology, writing original draft

AZ - Investigation, writing-review and editing

$\mathrm{AH}$ - Formal analysis, investigation, resources

OC - Forma analysis, resources, supervision

HS - Rsources, supervision

$\mathrm{CC}$ - Methodology, writing-review and editing

BG - Formal analysis, writing original draft

AS - Conceptualization, investigation, methodology, writing-review and editing

\section{Conflicts of Interest}

The authors declare no conflict of interest.

\section{References}

1. Alba AC, Bain E, Ng N, Stein M, Brien KO. Complications after Heart Transplantation: Hope for the Best, but Prepare for the Worst. Int J Transplant Res Med. 2016;2(2):2:022. DOI: 10.23937/2572-4045.1510022

2. Szymanska S, Grajkowska W, Sobieszczanska-Malek M, Zielinski T, Pyzlak M, Pronicki M. Prevalence of the Quilty effect in endomyocardial biopsy of patients after heart transplantation - from cellular rejection to antibody-mediated rejection? Pol J Pathol. 2016;67(3):21620. DOI: $10.5114 /$ pjp. 2016.63772

3. Hunt SA, Haddad F. The changing face of heart transplantation. J Am Coll Cardiol. 2008;52(8):587-98. DOI: $10.1016 /$ j.jacc.2008.05.020 
4. Cipullo R, Finger MA, Rossi Neto JM, Contreras $\mathrm{CM}$, Poltronieri NV, Zamorano Mde M, et al. Vasculitides and eosinophils in endomyocardial biopsies as rejection predictors in heart transplantation. Arq Bras Cardiol. 2011;97(2):163-70. DOI: 10.1590/S0066782X2011005000069

5. Mozaffari K, Bakhshandeh H, Amin A, Naderi N, Taghavi S, Ojaghi-Haghighi Z, et al. Diagnostic Pitfalls and Challenges in Interpretation of Heart Transplantation Rejection in Endomyocardial Biopsies With Focus on our Experience. Res Cardiovasc Med. 2014;3(1):e13986. DOI: 10.5812/cardiovascmed.13986

6. Zakliczynski M, Nozynski J, Konecka-Mrowka D, Pyka L, Trybunia D, Swierad M, et al. Quilty effect correlates with biopsy-proven acute cellular rejection but does not predict transplanted heart coronary artery vasculopathy. J Heart Lung Transplant. 2009;28(3):255-9. DOI: 10.1016/j.healun.2008.12.011

7. Chu KE, Ho EK, de la Torre L, Vasilescu ER, Marboe CC. The relationship of nodular endocardial infiltrates (Quilty lesions) to survival, patient age, anti-HLA antibodies, and coronary artery disease following heart transplantation. Cardiovasc Pathol. 2005;14(4):219-24. DOI: 10.1016/j.carpath.2005.03.009

8. Rigol M, Solanes N, Sionis A, Galvez C, Martorell J, Rojo I, et al. Effects of cyclosporine, tacrolimus and sirolimus on vascular changes related to immune response. J Heart Lung Transplant. 2008;27(4):416-22. DOI: 10.1016/j.healun.2008.01.006

9. Joong A, Richmond ME, Stack KO, Rodriguez RJ, McAllister JM, Zuckerman WA, et al. The Quilty Effect in Pediatric Heart Transplant Recipients. The Journal of Heart and Lung Transplantation. 35(4):S75. DOI: 10.1016/j.healun.2016.01.204

10. Cho H, Choi JO, Jeon ES, Kim JS. Quilty Lesions in the Endomyocardial Biopsies after Heart Transplantation. J Pathol Transl Med. 2019;53(1):50-6. DOI: 10.4132/ jptm.2018.11.30

11. Marboe CC, Billingham M, Eisen H, Deng MC, Baron $\mathrm{H}$, Mehra M, et al. Nodular endocardial infiltrates (Quilty lesions) cause significant variability in diagnosis of ISHLT Grade 2 and 3A rejection in cardiac allograft recipients. J Heart Lung Transplant. 2005;24(7 Suppl):S219-26. DOI: 10.1016/j.healun.2005.04.001

12. Kobashigawa J, Wener L, Johnson J, Currier JW, Yeatman L, Cassem J, et al. Longitudinal study of vascular remodeling in coronary arteries after heart transplantation. J Heart Lung Transplant. 2000;19(6):546-50. DOI: 10.1016/S1053-2498(00)00100-5

13. Minicucci MF, Zornoff LAM. VEGFR-2: One of Pioglitazone's Signaling Pathways in the Heart. Arq Bras Cardiol. 2018;111(2):170-1. DOI: 10.5935/ abc. 20180147
14. Waltenberger J, Kranz A, Beyer M. Neovascularization in the human heart is associated with expression of VEGF-A and its receptors Flt-1 (VEGFR-1) and KDR (VEGFR-2). Results from cardiomyopexy in ischemic cardiomyopathy. Angiogenesis. 1999;3(4):345-51. DOI: 10.1023/A:1026585900398

15. Ramzy D, Rao V, Brahm J, Miriuka S, Delgado D, Ross HJ. Cardiac allograft vasculopathy: a review. Can J Surg. 2005;48(4):319-27.

16. Patel JK, Kobashigawa JA. Cardiac transplant experience with cyclosporine. Transplant Proc. 2004;36(2 Suppl):323S-30S. DOI: 10.1016/j.transproceed.2004.01.039

17. Maravić-Stojković V, Stojković B, Perić M. Modern immunosuppressive agents after heart transplantation. Curr Trend Cardiol. 2017;1(2):41-8.

18. Kobashigawa JA, Miller LW, Russell SD, Ewald GA, Zucker MJ, Goldberg LR, et al. Tacrolimus with mycophenolate mofetil (MMF) or sirolimus vs. cyclosporine with MMF in cardiac transplant patients: 1-year report. Am J Transplant. 2006;6(6):1377-86. DOI: 10.1111/j.1600-6143.2006.01290.x

19. Lopez Garcia-Gallo C, Garcia Fadul C, Laporta R, Portero F, Millan I, Ussetti P. Cytomegalovirus Immunoglobulin for Prophylaxis and Treatment of Cytomegalovirus Infection in the (Val)Ganciclovir Era: A Single-Center Experience. Ann Transplant. 2015;20:6616. DOI: 10.12659/AOT.894694

20. Luckraz H, Charman SC, Wreghitt T, Wallwork J, Parameshwar J, Large SR. Does cytomegalovirus status influence acute and chronic rejection in heart transplantation during the ganciclovir prophylaxis era? J Heart Lung Transplant. 2003;22(9):1023-7. DOI: 10.1016/ S1053-2498(02)01185-3

21. Bolovan-Fritts CA, Trout RN, Spector SA. High T-cell response to human cytomegalovirus induces chemokine-mediated endothelial cell damage. Blood. 2007;110(6):1857-63. DOI: 10.1182/ blood-2007-03-078881

22. Frantzeskaki FG, Karampi ES, Kottaridi C, Alepaki M, Routsi C, Tzanela M, et al. Cytomegalovirus reactivation in a general, nonimmunosuppressed intensive care unit population: incidence, risk factors, associations with organ dysfunction, and inflammatory biomarkers. J Crit Care. 2015;30(2):276-81. DOI: 10.1016/j. jcrc.2014.10.002

23. Liu XF, Wang X, Yan S, Zhang Z, Abecassis M, Hummel M. Epigenetic control of cytomegalovirus latency and reactivation. Viruses. 2013;5(5):1325-45. DOI: $10.3390 / \mathrm{v} 5051325$

24. Barge-Caballero E, Almenar-Bonet L, Crespo-Leiro MG, Brossa-Loidi V, Rangel-Sousa D, Gomez-Bueno $\mathrm{M}$, et al. Preoperative Toxoplasma gondii serostatus 
does not affect long-term survival of cardiac transplant recipients. Analysis of the Spanish Heart Transplantation Registry. Int J Cardiol. 2018;250:183-7. DOI: 10.1016/j.ijcard.2017.09.215

25. Kittleson MM, Kobashigawa JA. Toxoplasma gondii exposure in the heart transplant recipient: good, bad, or indifferent? Transplantation. 2013;96(12):1025. DOI: 10.1097/TP.0b013e3182a92769

26. Vitrone M, Iossa D, Rinaldi L, Pafundi PC, Molaro R,
Parrella A, et al. Hepatitis B virus reactivation after heart transplant: Incidence and clinical impact. J Clin Virol. 2017;96:54-9. DOI: 10.1016/j.jcv.2017.09.011

27. Iossa D, Vitrone M, Liotti A, Portella G, Durante-Mangoni E, Zampino R. Hepatitis B core-related antigen to detect hepatitis $\mathrm{B}$ virus (HBV) reactivation in heart transplant recipients with past HBV infection: A pilot study. Clin Transplant. 2019;33(6):e13574. DOI: $10.1111 /$ ctr.13574 